УДК 538.9:536.6

\title{
ТЕПЛОФИЗИЧЕСКИЕ СВОЙСТВА НИЗКОТЕПЛОПРОВОДНЫХ ПОЛИМЕРНЫХ НАНОКОМПОЗИТОВ ДЛЯ ЭЛЕМЕНТОВ ЭНЕРГЕТИЧЕСКОГО ОБОРУДОВАНИЯ
}

Долинський А.А. ${ }^{1}$, академик НАН Украины Фиалко Н.М. ${ }^{1}$, д.Т.н., Динжос Р.В. ${ }^{2}$, канд. физ.-мат. наук, Навродская Р.А. ${ }^{,}$, канд. техн. наук

${ }^{1}$ Институт технической теплофизики НАН Украинь,, ул. Желябова, 2 а, Киев, 03680, Украина

${ }^{2}$ Николаевский национальный университет им. В.А.Сухомлинского, ул. Никольская, 24, Николаев, 54030, Украина

Наведено результати експериментальних досліджень теплофізичних, структурних i механічних характеристик низькотеплопровідних полімерних нанокомпозитів, орієнтованих на виготовлення трубопроводів різних систем, захисних теплоізоляційних шарів енергетичного обладнання тощо. Представлено дані методичних досліджень щодо аналізу можливостей застосування для прогнозування теплопровідних властивостей нанокомпозитів ряду методів теорії ефективного середовища і теорії перколяції.

Библ. 13 , рис. 4 , табл. 3.
Приведены результаты экспериментальных исследований теплофизических, структурных и механических характеристик низкотеплопроводных полимерных нанокомпозитов, ориентированных на изготовление трубопроводов различных систем, защитных теплоизоляционных слоев энергетического оборудования и пр. Представлены данные методических исследований, касающихся анализа возможностей применения для прогнозирования теплопроводящих свойств нанокомпозитов ряда методов теории эффективной среды и теории перколяции.
The results of experimental studies of thermal, structural and mechanical characteristics of the low thermal conductivity of polymer nanocomposites, focused on the production of pipes of different systems, protective thermal insulation layers of power equipment and so on are presented. The data of methodological studies on the analysis of possible of applications to predict the heat conducting properties of the nanocomposites a number of methods of effective medium theory, and percolation theory are given.

Ключевые слова: низкотеплопроводные полимерные нанокомпозиты, теоретические модели теплопроводности, теплофизические свойства.

$C$ - фактор влияния композита;

$c_{p}$ - удельная массовая теплоемкость, Дж/(кг·К);

$k$ - показатель степени в уравнениях Киркпатрика и Маклахлана;

$p$ - массовая доля наполнителя, $0<p<1$;

$p_{c}$ - значение $\mathrm{p}$, отвечающее порогу перколяции;

$T$ - температура, К;

$q$ - показатель степени в уравнениях Киркпатрика и Маклахлана;

$X$ - степень кристалличности композита, \%;

$\lambda_{1}, \lambda_{2}-$ коэффициент теплопроводности материа-

\section{Введение}

Среди перспективных областей применения полимерных нанокомпозиционных материалов особо выделяется использование их низкотепло- ла матрицы и наполнителя, Вт/(м К);

$\lambda^{\prime}, \lambda^{\prime \prime}-$ коэффициент теплопроводности комозита

в непосредственной близости слева $\mathrm{p}_{\text {c- }}$ и справа $\mathrm{p}_{\mathrm{c}^{+}}$от порога перколяции;

$\lambda_{э ф} \lambda$ - коэффициент теплопроводности композита, Вт/(м К);

$\chi_{k}^{2}, \chi_{q}^{2}-$ значение дисперсии для уравнений Киркпатрика и Маклахлана;

$\omega-$ массовая доля наполнителя, \%;

$\omega_{c}$ - значение $\omega$, отвечающее порогу перколяции; УНТ - углеродные нанотрубки.

проводных модификаций для создания трубопроводов различных систем, защитной теплоизоляции энергетического оборудования и т.д. [1-5].

В ряде практически важных ситуаций к низ- 
котеплопроводным композиционным материалам для элементов энергетических систем наряду с требованиями в отношении их теплофизических характеристик предъявляются также требования в части механических свойств. Последнее связано с необходимостью обеспечения конструкционной прочности данных элементов. Ввиду этого актуальной является задача разработки низкотеплопроводных полимерных нанокомпозитов с улучшенными механическими свойствами для изготовления деталей энергетических систем и исследования их теплофизических, структурных и механических характеристик.

Что касается исследований теплофизических свойств получаемых композиционных материалов, то здесь представляет интерес рассмотрение различных теоретических методов их прогнозирования в широком диапазоне изменения составляющих компонентов. Так, при определении коэффициентов теплопроводности нанокомпозитов возможно использование целого ряда методов из класса методов теории эффективной среды и теории перколяции [6-10]. Это обуславливает актуальность выбора из существующих методов тех из них, которые дают наиболее адекватное описание поведения теплопроводящих свойств полимерных композитов.

\section{Постановка задачи и методика проведения исследований.}

Данная статья посвящена исследованию теплофизических свойств, структурных характеристик и модуля Юнга низкотеплопроводных полимерных нанокомпозитов на основе полиэтилена и полипропилена, которые ориентированы на изготовление трубопроводов различного назначения (воздухо-, водо-, масло-, топливопроводов и пр.). В методическом плане в задачу исследования входило проведение сравнительного анализа теоретических моделей теплопроводности.

В ходе исследований использовался полиэтилен высокого давления и низкой плотности. В качестве наполнителей применялись УНТ и наночастицы пирогенного аэросила $\mathrm{SiO}_{2}$. Описание основных характеристик используемых УНТ дано в [11]. Наночастицы аэросила кремния (Aerosil 1380, Degussa Co, Германия) имели диаметр 40 нм. Массовая доля наполнителей в по- лимерных нанокомпозитах изменялась от 0,1 до $3 \%$.

В качестве метода получения нанокомпозитов применялся метод, базирующийся на смешении компонентов в расплаве полимера с использованием экструдера при дальнейшем придании композиту необходимой формы способом горячего прессования [12]. Экспериментальные методики определения теплофизических свойств разрабатываемых композитов приведены в [11]. Для определения модуля Юнга использовался метод деформационной калориметрии.

Теоретическое описание концентрационного поведения коэффициентов теплопроводности рассматриваемых полимерных нанокомпозитов основывалось на использовании двух классов моделей теплопроводности. Первому из них отвечали модели теории эффективной среды, второму - модели теории перколяции.

В работе рассмотрению подлежали две модели теории эффективной среды - модель Максвелла и модифицированная модель Бруггемана [6-8]. Согласно модели Максвелла эффективная тепло-

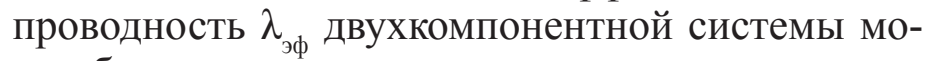
жет быть определена по зависимости

$\lambda_{\text {э }}=\lambda_{1}\left(\frac{\lambda_{2}+2 \lambda_{1}-2 p\left(\lambda_{1}-\lambda_{2}\right)}{\lambda_{2}+2 \lambda_{1}+p\left(\lambda_{1}-\lambda_{2}\right)}\right)$.

Данная модель базируется на представлении о хаотическом внедрении в изотропную среду сферических частиц, располагающихся на достаточно большом расстоянии друг от друга так, что их взаимодействием можно пренебречь.

Модифицированная модель Бруггемана относится к более поздним разработкам [7]. В ней принимается во внимание целый ряд факторов, таких как компактная упаковка и взаимодействие между частицами, форма частиц, их дисперсность, межфазное термическое сопротивление и пр. В соответствии с данной моделью выражение для нахождения эффективной теплопроводности гетерогенной системы имеет вид

$1-p=\frac{\lambda_{2}-\lambda_{\ni \phi}}{\lambda_{2}-\lambda_{1}}\left(\frac{c \lambda_{1}}{\lambda_{\ni \phi}}\right)^{\frac{1}{3}}$

Приведенные модели, как и прочие модели теории эффективной среды, основываются на 
положении о «парциальных» вкладах матрицы и наполнителя. В них не учитываются процессы агрегации частиц наполнителя, в результате которых образовываются теплопроводящие каналы, обусловливающие эффекты скачкообразного повышения теплопроводности композита. В моделях теории эффективной среды не принимаются также во внимание такие явления, как изменение теплофизических свойств полимера на границе «полимер-частица», разрыхление полимеров с образованием пор и пр. [8].

От указанных недостатков свободны модели теории перколяции. Согласно перколяционной модели Киркпатрика эффективная теплопроводность полимерного композита может быть представлена зависимостью[9]

$\lambda_{\text {эф }}=\left\{\begin{array}{cc}\lambda^{\prime}\left(p_{c}-p\right)^{-q} & \text { nри } p<p_{c}, \\ \lambda^{\prime \prime}\left(p-p_{c}\right)^{k} & \text { nри } p>p_{c} .\end{array}\right.$

В соответствии с перколяционной моделью Маклахлана выражение, описывающее зависимость коэффициента теплопроводности композита от содержания наполнителя, имеет вид [10]

$$
\lambda=\left\{\begin{array}{lll}
\lambda^{\prime}\left(\frac{p_{c}}{p_{c}-p}\right)^{q} & \text { npu } & p<p_{c}, \\
\lambda^{\prime \prime}\left(\frac{p-p_{c}}{1-p_{c}}\right)^{k} & \text { npu } & p>p_{c} .
\end{array}\right.
$$

В рамках данной работы ставилась задача сопоставления приведенных моделей теплопроводности для рассматриваемых композиционных материалов.

\section{Результаты исследований и их анализ}

а) Теоретические модели теплопроводности полимерных композитов.

Остановимся вначале на рассмотрении результатов исследований, посвященных анализу возможностей использования различных теоретических моделей теплопроводности гетерогенных систем. На рис. 1, 2 представлены концентрационные зависимости коэффициентов теплопроводности получаемых полимерных нанокомпозитов по данным экспериментов и различных теоретических моделей.

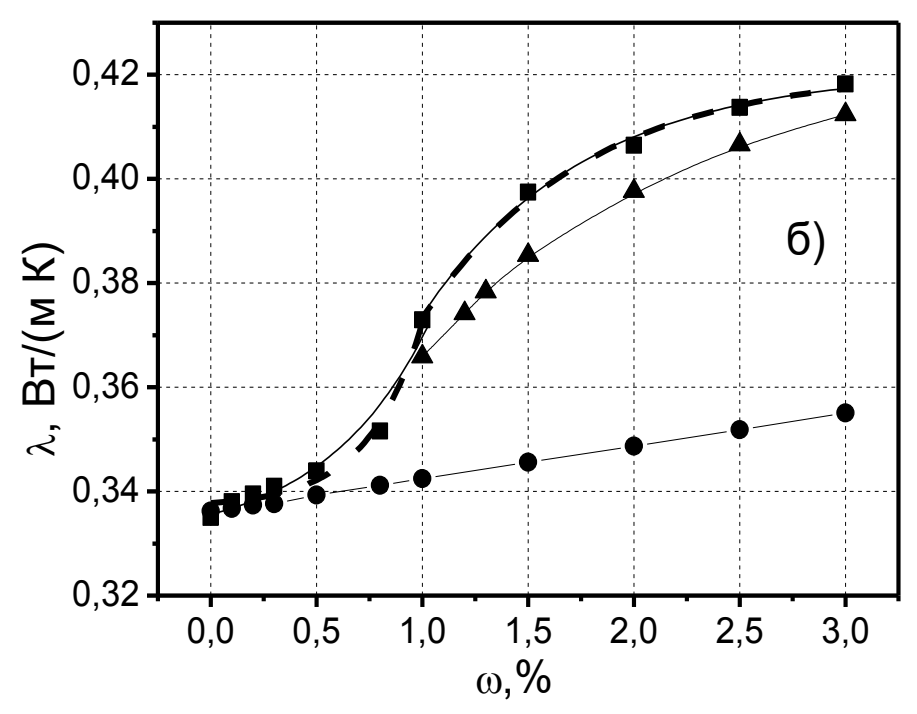

Рис. 1. Концентрационная зависимость коэффициента теплопроводности полимерных нанокомпозитов на основе полиэтилена, наполненного УНТ (a)

и нанодисперсными частицами аэросила (б);

1 - результаты эксперимента; 2-5 - данные теоретических моделей:

2, 3 -модель Максвелла и модифицированная модель Бругzемана

из класса моделей эффективной среды;

4, 5 -модель Киркпатрика и Маклахлана из класса перколяционных моделей. 
Как следует из приведенных данных, модели теории эффективной среды - модель Максвелла и модифицированная модель Бруггемана - адекватно описывают концентрационное поведение коэффициента теплопроводности только в определенных диапазонах содержания наполнителя. Так, модель Максвелла удовлетворительно согласуется с результатами экспериментальных исследований лишь при весьма малых массовых долях наполнителей, не превышающих значение порога перколяции $\omega_{c}$. В области же $\omega>\omega_{\text {с дан- }}$ ная модель, как очевидно, неприменима. Здесь величины $\lambda$, полученные в эксперименте, могут превышать значения, отвечающие модели Максвелла, более чем на 45 \%.

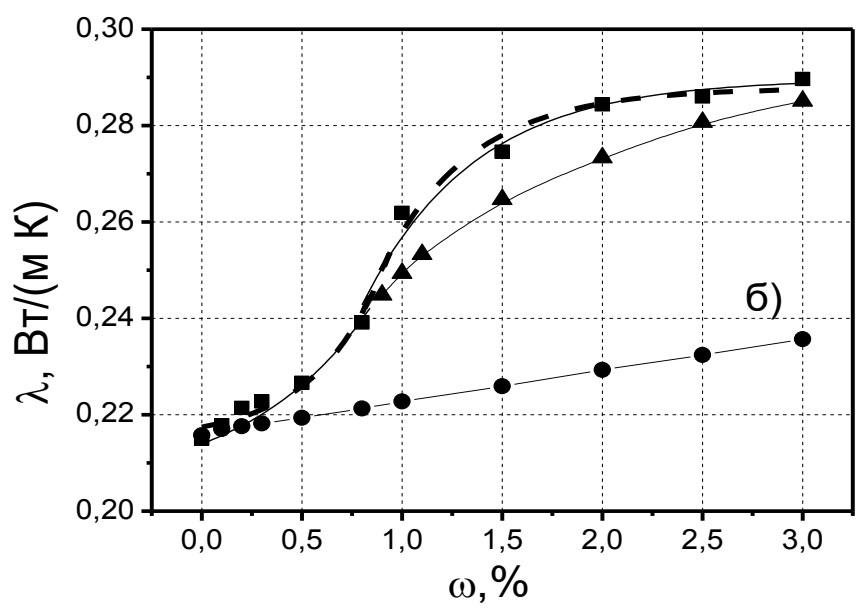

Рис. 2. Зависимость коэффициента теплопроводности полимерных нанокомпозитов на основе полипропилена, наполненного УНТ (а) и нанодисперсными частицами аэросила (б); 1 -результаты эксперимента; 2 - 5 - данные теоретических моделей:

2, 3 -модель Максвелла и модифицированная модель Бругzемана из класса моделей эффективной среды; 4, 5 -модель Киркпатрика и Маклахлана из класса перколяционных моделей.

Что касается модифицированной модели Бруггемана, то она, напротив, не может быть использована при массовых долях наполнителя меньше перколяционного порога, однако применима для значений $\omega$, превышающих данный порог. При этом для разрабатываемых композитов максимальные расхождения экспериментальных и расчетных величин $\lambda$ в области $\omega>\omega_{\text {c }}$ находится в пределах $3 \ldots .8 \%$.

Таким образом, рассмотренные модели теории эффективной среды применительно к анализируемой ситуации могут иметь лишь ограниченное использование.

Как показали выполненные исследования, модели теории перколяции - модель Киркпатрика и модель Маклахлана - дают адекватное описание концентрационного поведения композитов и отражают эффект резкого изменения теплопроводности по достижении перколяционного порога.
Причем отклонения результатов экспериментов и данных указанных моделей являются весьма незначительными.

В табл. 1 представлены найденные параметры уравнений, отвечающие двум рассматриваемым моделям. Как видно, при использовании модели Маклахлана обеспечиваются несколько меньшие расхождения экспериментальных и расчетных данных (сопоставьте значения дисперсии для уравнений (3) и (4) в табл. 1). При этом для указанной модели лучшее согласование сопоставляемых результатов имеет место в случае композитов на основе полиэтилена по сравнению с композитами на основе полипропилена. Для обеих полимерных матриц меньшие отклонения результатов расчетов от экспериментальных данных наблюдаются при наполнении полимеров УНТ, чем при их наполнении аэросилом.

Резюмируя выполненный анализ перколяци- 
онных моделей, необходимо отметить, что они характеризуются высокой точностью описания зависимости коэффициентов теплопроводности нанокомпозитов от массовой доли наполнителя. Это связано с тем, что в данных моделях по сравнению с моделями теории эффективной среды учитывается целый ряд дополнительных факторов, таких как формирование проводящих перко- ляционных структур, изменение характеристик полимера вблизи частиц наполнителя, образование системы пор и т.д. Важным является также то обстоятельство, что рассмотренные перколяционные модели ввиду их высокой точности могут успешно использоваться для прогнозирования свойств полимерных композитов в широком диапазоне изменения их состава.

Таблица 1. Параметры уравнений (3), (4) для различных полимерных матриц и нанодисперсных наполнителей

\begin{tabular}{|c|c|c|c|c|c|c|c|c|c|}
\hline \multicolumn{7}{|c|}{ Модель Киркпатрика (уравнение (3)) } & \multicolumn{7}{c|}{ Модель Маклахлана (уравнение (4)) } \\
\hline$k$ & $\chi_{k}^{2}, 10^{-5}$ & $q$ & $\chi_{q}^{2}, 10^{-5}$ & $p_{c} \times 100, \%$ & $k$ & $\chi_{k}^{2}, 10^{-5}$ & $q$ & $\chi_{q}^{2}, 10^{-5}$ & $p_{c} \times 100, \%$ \\
\hline \multicolumn{8}{|c|}{ Матрица - полиэтилен, наполнитель - УНТ } \\
\hline 0,013 & 0,9 & 0,042 & 6,0 & 0,79 & 0,02 & 0,6 & 0,047 & 0,8 & 0,78 \\
\hline \multicolumn{8}{|c|}{ Матрица - полиэтилен, наполнитель - аэросил } \\
\hline 0,01 & 21,0 & 0,018 & 16,0 & 0,99 & 0,012 & 6,0 & 0,022 & 5,0 & 0,96 \\
\hline \multicolumn{8}{|c|}{ Матрица - полипропилен, наполнитель - УНТ } \\
\hline 0,02 & 3,0 & 0,062 & 90,0 & 0,58 & 0,021 & 2,0 & 0,066 & 4,0 & 0,57 \\
\hline \multicolumn{8}{|c|}{ Матрица - полипропилен, наполнитель - аэросил } \\
\hline 0,013 & 10,0 & 0,049 & 30,0 & 0,84 & 0,014 & 8,0 & 0,053 & 6,0 & 0,81 \\
\hline
\end{tabular}

б) Теплофизические, структурные и механические свойства низкотеплопроводных полимерных нанокомпозитов.

Как уже отмечалось, низкотеплопроводные полимерные композиты в настоящее время завоевывают все большую популярность в качестве материалов для создания трубопроводов различного назначения, защитных теплоизоляционных слоев энергетического оборудования и т.д. Что касается трубопроводов, то к полимерным композитам для их изготовления предъявляется целый ряд требований, основные из которых касаются, во-первых, величины коэффициента теплопроводности и диапазона рабочих температур материала, и, во-вторых, значения модуля упругости при растяжении (модуля Юнга). При этом в зависимости от назначения и условий эксплуатации трубопроводов данные требования оказываются существенно различными. Так, для трубопроводов, транспортирующих в безнапорном режиме сточные воды при максимальной температуре постоянных стоков 70 들, модуль Юнга композита должен быть не меньше 1,15 ГПа, для напорных газовых и водопроводных труб - 0,2 ГПа [13]. Относительно же значений коэффициента теплопроводности, то здесь требования определяются, главным образом, необходимостью обеспечения при эксплуатации тех или иных трубопроводов заданного уровня теплопотерь.

Ниже на примере нанокомпозитов на основе полиэтилена и полипропилена, предназначенных для создания различных трубопроводов, показана возможность существенного повышения их модуля Юнга по сравнению с ненаполненными полимерами при относительно небольшом повышении коэффициентов теплопроводности. На рис. 3 по результатам проведенных экспериментальных исследований представлены соответствующие концентрационные зависимости. Как видно, характер изменения величины $\lambda$ и $\mathrm{E}$ в целом аналогичен. То есть имеет место тенденция к увеличению значений $\lambda$ и Е при возрастании массовой доли наполнителя. Что же касается эффектов скачкообразного изменения этих вели- 
чин, то они связаны с образованием из частиц наполнителя проводящих перколяционных структур. Обращает на себя внимание тот факт, что указанные скачкообразные изменения модуля Юнга наблюдаются при несколько меньших значениях массовой доли наполнителя, чем соответствующие изменения коэффициента

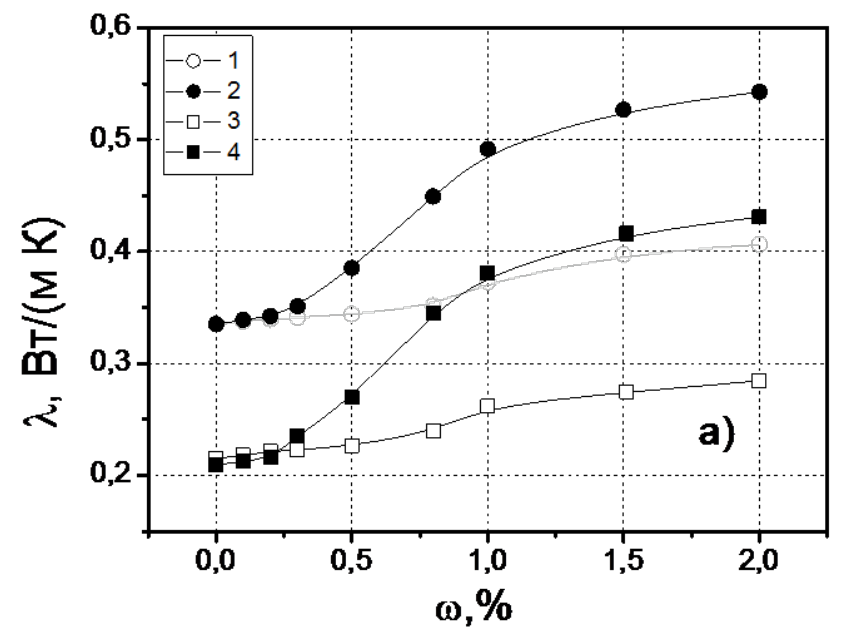

теплопроводности. (например, для полиэтилена, наполненного УНТ, резкому изменению величины $\lambda$ отвечает значение $\omega=0,78 \%$, а модуля Юнга $-\omega=0,60 \%$ ). Это свидетельствует о большей чувствительности механических характеристик к формированию перколяционных кластеров и сеток.

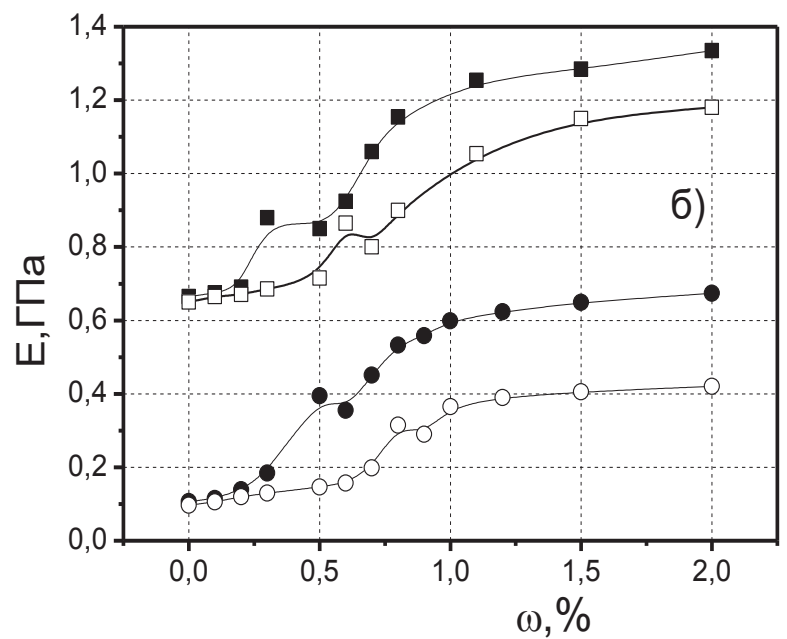

Рис. 3. Концентрационные зависимости коэффициента теплопроводности (а) и модуля Юнга (б) композитов на основе полиэтилена $(1,2)$ и полипропилена $(3,4)$, наполненных УНТ $(2,4)$ и наночастицами аэросила $(1,3)$.

Относительно значений массовой концентрации, соответствующих скачкообразному изменению величин $\lambda$ и Е, следует также отметить, что они оказываются меньшими при наполнении полимеров УНТ, чем при их наполнении аэросилом. К тому же эти значения при использовании обоих рассматриваемых наполнителей сдвигаются в область больших величин $\omega$ для композитов на основе полипропилена в сравнении с композитами на основе полиэтилена.

Что касается концентрационной зависимости модуля Юнга, то здесь в первую очередь необходимо указать на то обстоятельство, что при относительно небольшом содержании наполнителей (до 2 \%) оказывается возможным существенное повышение величины Е. Так, для полиэтилена, наполненного УНТ, значение Е возрастает более чем в 6 раз при увеличении $\omega$ от 0 до $2 \%$.

Как видно из рис. 3, б, для композитов на основе полипропилена величины Е выше, чем для композитов на основе полиэтилена во всем рассматриваемом диапазоне изменения как очевидно, связано с более высоким значением модуля Юнга для ненаполненного полипропилена по сравнению с полиэтиленом. А именно, для полипропилена это значение равно 0,665 ГПа, а для полиэтилена - только 0,106 ГПа. Согласно полученным данным модуль Юнга для композитов, наполненных УНТ, превышает соответствующие значения для композитов, наполненных аэросилом. Причем эти отличия в целом оказываются несколько большими для композитов на основе полиэтилена. Например, при $\omega=2 \%$ расхождение значений Е, отвечающих УНТ и аэросилу, для полипропилена составляют 0,150 ГПа, а для полиэтилена - 0,253 ГПа.

Как показали полученные данные, в рассматриваемых условиях наполнение полимеров не приводит к значительному росту коэффициентов теплопроводности (см. рис. 3, $a$ ). При этом большее повышение $\lambda$ наблюдается при прочих равных условиях для композитов на основе полиэтилена. Это обусловлено более высокими значениями $\lambda$ для ненаполненного полиэтилена по 
сравнению с полипропиленом. Что же касается нанодисперсных наполнителей, то применение углеродных нанотрубок приводит к существенно большему возрастанию $\lambda$, чем применение аэросила.

В табл. 2, 3 представлены результаты экспериментальных исследований по определению температурной зависимости удельной массовой теплоемкости для получаемых полимерных композитов. Согласно приведенным данным температура фазового перехода для этих композиционных материалов практически не зависитот массовой доли наполнителя. То есть диапазон их рабочих температур отвечает соответствующему диапазону для полимерных матриц.

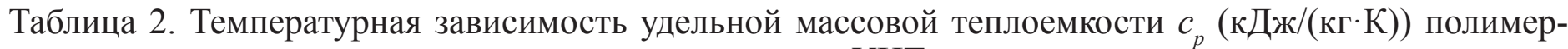
ного композита на основе полиэтилена, наполненного УНТ и нанодисперсными частицами аэросила

\begin{tabular}{|c|c|c|c|c|c|c|c|c|c|c|c|}
\hline \multirow{2}{*}{$\begin{array}{l}\text { Напол- } \\
\text { нитель }\end{array}$} & \multirow{2}{*}{$\begin{array}{c}\text { Содержание } \\
\text { наполнителя, } \\
\text { \% }\end{array}$} & \multicolumn{10}{|c|}{$\mathrm{T}, \mathrm{K}$} \\
\hline & & 320 & 380 & 385 & 390 & 395 & 400 & 405 & 410 & 415 & 420 \\
\hline \multirow{3}{*}{ УНТ } & 0,3 & 1,78 & 2,76 & 3,16 & 3,62 & 4,98 & 6,96 & 12,9 & 15,3 & 5,01 & 2,51 \\
\hline & 2 & 1,72 & 2,67 & 3,04 & 3,48 & 4,79 & 6,71 & 12,4 & 14,8 & 4,83 & 2,42 \\
\hline & 3 & 1,73 & 2,65 & 3,03 & 3,46 & 4,76 & 6,64 & 12,2 & 14,6 & 4,79 & 2,41 \\
\hline \multirow{3}{*}{ Аэросил } & 0,3 & 1,77 & 2,76 & 3,17 & 3,63 & 5,01 & 7,01 & 12,9 & 15,5 & 5,04 & 2,52 \\
\hline & 2 & 1,78 & 2,74 & 3,14 & 3,57 & 4,90 & 6,82 & 12,6 & 14,9 & 4,94 & 2,50 \\
\hline & 3 & 1,79 & 2,73 & 3,11 & 3,64 & 4,85 & 6,74 & 12,3 & 14,7 & 4,88 & 2,49 \\
\hline
\end{tabular}

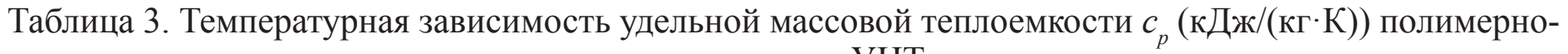
го композита на основе полипропилена, наполненного УНТ и нанодисперсными частицами аэросила

\begin{tabular}{|c|c|c|c|c|c|c|c|c|c|c|c|}
\hline \multirow{2}{*}{$\begin{array}{l}\text { Напол- } \\
\text { нитель }\end{array}$} & \multirow{2}{*}{$\begin{array}{c}\text { Содержание } \\
\text { наполнителя, } \\
\text { \% }\end{array}$} & \multicolumn{10}{|c|}{$\mathrm{T}, \mathrm{K}$} \\
\hline & & 300 & 360 & 420 & 425 & 430 & 435 & 440 & 442 & 445 & 450 \\
\hline \multirow{3}{*}{ УНТ } & 0,3 & 1,75 & 2,27 & 3,56 & 4,10 & 4,88 & 5,81 & 6,71 & 6,79 & 6,16 & 3,11 \\
\hline & 2 & 1,82 & 2,26 & 3,36 & 3,82 & 4,49 & 5,29 & 6,05 & 6,11 & 5,58 & 2,97 \\
\hline & 3 & 1,88 & 2,28 & 3,29 & 3,72 & 4,33 & 5,05 & 5,75 & 5,81 & 5,32 & 2,93 \\
\hline \multirow{3}{*}{ Аэросил } & 0,3 & 1,75 & 2,28 & 3,62 & 4,17 & 4,98 & 5,94 & 6,87 & 6,95 & 6,31 & 3,15 \\
\hline & 2 & 1,82 & 2,31 & 3,55 & 4,07 & 4,82 & 5,69 & 6,57 & 6,64 & 6,05 & 3,11 \\
\hline & 3 & 1,91 & 2,38 & 3,56 & 4,06 & 4,77 & 5,61 & 6,45 & 6,52 & 5,95 & 3,14 \\
\hline
\end{tabular}

Полученные экспериментальные зависимости удельной массовой теплоемкости композитов от температуры послужили также основой для определения одной из важнейших структурных характеристик полимерных композитов - степени их кристалличности [12]. Как видно из данных, приведенных на рис. 4, понижение степени кристалличности при увеличении массовой доли наполнителя оказывается несколько более значительным для композитов на основе полипропилена. Например, это понижение в рассматриваемом диапазоне изменения $\omega$ в случае наполнения полимеров УНТ составляет для матрицы из полиэтилена 7,6 \%, а для матрицы из полипропилена $-11,8$ \%. При этом для обеих матриц меньшее падение степени кристалличности $\mathrm{X}$ наблюдается при их наполнении аэросилом. Последнее, как очевидно, связано с меньшей эффективностью образования перколяционных структур наночастицами аэросила в сравнении с УНТ. 

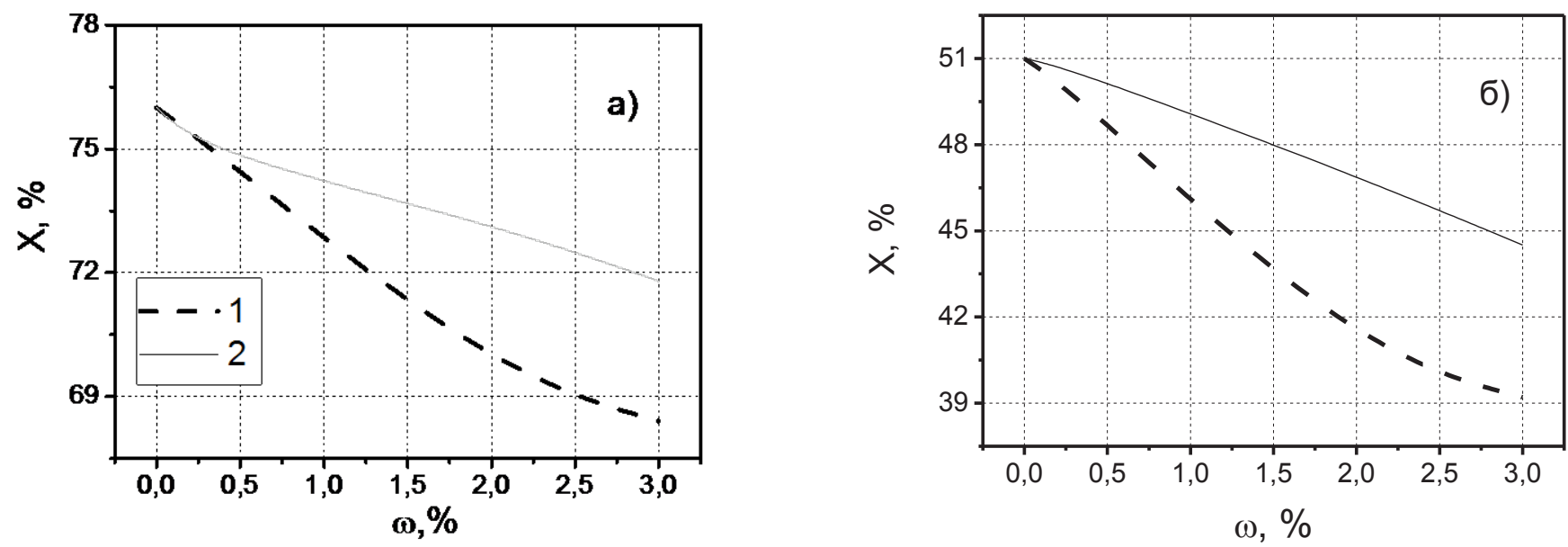

Рис. 4. Зависимость степени кристалличности полиэтилена (а) и полипропилена (б) от массовой доли наполнителя для композитов, содерэнацих УНТ (1) и аэросил (2).

\section{Выводы}

1. На основе экспериментальных исследований установлена возможность получения низкотеплопроводных полимерных нанокомпозитов с улучшенными механическими характеристиками для трубопроводов различного назначения. В частности, показано, что для нанокомпозитов на основе полиэтилена или полипропилена, наполненных УНТ либо нанодисперсными частицами аэросила, при массовой доле последних до $2 \%$ имеет место:

a) относительно незначительное повышение коэффициентов теплопроводности (до $\lambda=$ $=0,54 \mathrm{BT} /($ м К $))$;

б) существенное возрастание модуля упругости при растяжении (до $\mathrm{E}=1,33$ ГПа).

2. Для разрабатываемых полимерных нанокомпозитов получены температурные зависимости их удельной массовой теплоемкости и на этой основе проведен анализ закономерностей изменения структурных характеристик данных материалов.

3. Выполнен комплекс методических исследований по анализу правомерности использования для прогнозирования теплопроводящих свойств рассматриваемых композитов различных моделей теплопроводности. Рассмотрены возможности применения ряда моделей теории эффективной среды и теории перколяции. Показано, что:

а) модели теории эффективной среды - мо- дель Максвелла и модифицированная модель Бруггемана - адекватно описывают концентрационное поведение коэффициента теплопроводности только в определенных диапазонах содержания наполнителя;

б) модели теории перколяции - модель Киркпатрика и модель Маклахлана - обеспечивают адекватное прогнозирование теплопроводящих свойств композитов во всем диапазоне изменения содержания наполнителя; при этом модель Маклахлана является несколько более предпочтительной.

\section{ЛИТЕРАТУРА}

1. Hachour $K$. Experiments and modeling of high-crystalline polyethylene yielding under different stress states / K. Hachour, F. Zanri, M. Nant-Abdelaziz, J.M. Gloaguen, M. Aberkane, J.M. Lefebvre. - International Journal of Plasticity. 2014. - Vol. 54. - P. 1-18.

2. Naganathan S. Nanotechnology in Civil Engineering - A Review / Sivakumar Naganathan, Charan Singh Jasbir Singh, Yim Wil Shen, Peng Eng Kiat, Sivadass Thiruchelvam. - Advanced Materials Research. - 2014. - Vol. 935. - P. 151-154.

3. Benyahia F. A Comparison Study of Bonded Composite Repairs of through-Wall Cracks in Pipes Subjected to Traction, Bending Moment and Internal Pressure / F. Benyahia, A. Albedah, B. Bachir Bouiadjra, M. Belhouari. - Advanced Materials Research. - 2015. - Vol. 1105. - P. 41-45. 
4. Suner S. Ultra high molecular weight polyethylene/graphene oxide nanocomposites: Thermal, mechanical and wettability characterisation / S. Suner, R. Joffe, J.L.Tipper, N. Emami. - Composites Part B. - 2015. - Vol. 78. P. 185-191.

5. Damodaran S. Monitoring the effect of chlorine on the ageing of polypropylene pipes by infrared microscopy / Subin Damodaran, Tobias Schuster, Karsten Rode, Abhishek Sanoria, Robert Brüll, Mirko Wenzel, Martin Bastian. - Polymer Degradation and Stability. - 2015. - Vol. 66 - P. 7-19.

6. Agari Y. Thermal Conductivity of a Polymer Composite Filled with Mixtures of Particles / Y. Agari, M. Tanaka, S. Nagai. - Journal of Applied Polymer Science. - 1987. - Vol. 34. - P. 1429-1437.

7. Lin C. Modeling and analysis of synergistic effect in thermal conductivity enhancement of polymer composites with hybrid filler / Lin Chen, Ying-Ying Sun, Jun Lin, Xiao-Ze Du, Gao-Sheng Wei, Shao-Jian He, Sergei Nazarenko. - International Journal of Heat and Mass Transfer. - 2015 - - Vol. 81. - P. 457-464.

8. Shen $M$. Thermal conductivity model of filled polymer composites / Ming-xia Shen, Yin-xin Cui, Jing He, and Yao-ming Zhang. - International Journal of Minerals, Metallurgy and Materials. - 2011. - Vol.
18, №5. - P. 623-631.

9. Kirkpatrick S. Percolation and Conduction / Scott Kirkpatrick. - Reviews of modern physics. 1973. - Vol. 45, №4. - P. 574-585.

10. McLachlan D.S. The correct modelling of the second order terms of the complex ACconductivity results for continuum percolation media, using a single phenomenological equation / D.S. McLachlan, C. Chiteme, W.D. Heiss, Junjie Wu. - Physica B. 2003. - Vol. 338. - P. 256-260.

11. Долинский А. А. Теплофизические свойства полимерных микро- и нанокомпозитов на основе поликарбоната / А. А. Долинский, Н. М. Фиалко, Р. В. Динжос, Р. А. Навродская // Промышленная теплотехника.- 2015. - №2. - С. 1219.

12. Долинский А. А. Влияние методов получения полимерных микро- и нанокомпозитов на их теплофизические свойства / А. А. Долинский, Н. М. Фиалко, Р. В. Динжос, Р. А. Навродская // Промышленная теплотехника.- 2015. - № 4. - С. 5-13.

13. Кацевман М. Применение концентратов минеральных наполнителей в производстве полимерных труб / Михаил Кацевман, Сергей Киселев, Игорь Айзинсов. - Полимерные трубы. 2011. - Vol. 31. - C. 31-37. 
THERMOPHYSICAL PROPERTIES OF LOW THERMAL CONDUCTIVITY OF POLYMER NANOCOMPOSITES FOR ELEMENTS OF THE POWER EQUIPMENT

\section{Dolinskiy A.A. ${ }^{\text {, Fialko N.M. }}{ }^{1}$, Dinzhos R.V. ${ }^{2}$, Navrodskaya R.A. ${ }^{1}$}

${ }^{1}$ Institute of Engineering Thermophysics, National Academy of Sciences of Ukraine, 2a, Zhelyabova str., Kyiv, 03680, Ukraine

${ }^{2}$ Nikolaev National University. named after V.A. Sukhomlinskiy, 24, Nikolska str., Mykolaev, 54030, Ukraine

The data of experimental studies of thermo physical, structural and mechanical characteristics of the low heat conductivity of polymer nanocomposites, oriented on the production of different systems pipes, protective heat-insulated layers of power equipment and so on are given. The results of a comparison of these characteristics for nanocomposites based on polyethylene and polypropylene filled with CNTs or nanoparticles aerosil are considered. The data of methodological studies relating to the analysis of applications possible of a number of methods of the effective medium theory and the percolation theory to predict the heat conducting properties of the nanocomposites are presented. The materials that define the area of adequate prediction of the heat conductivity values of composites based on concerned theoretical methods.

Key words: low heat conductivity of polymer nanocomposites, theoretical model of the heat conductivity, thermo physical properties.

References 13, tabl. 3, fig. 4.

1. Hachour $K$. Experiments and modeling of high-crystalline polyethylene yielding under different stress states / K. Hachour, F. Zanri, M. Nant-Abdelaziz, J.M. Gloaguen, M. Aberkane, J.M. Lefebvre. - International Journal of Plasticity. 2014. - Vol. 54. - P. 1-18.

2. Naganathan S. Nanotechnology in Civil Engineering - A Review / Sivakumar Naganathan, Charan Singh Jasbir Singh, Yim Wil Shen, Peng Eng
Kiat, Sivadass Thiruchelvam. - Advanced Materials Research. - 2014. - Vol. 935. - P. 151-154.

3. Benyahia F. A Comparison Study of Bonded Composite Repairs of through-Wall Cracks in Pipes Subjected to Traction, Bending Moment and Internal Pressure / F. Benyahia, A. Albedah, B. Bachir Bouiadjra, M. Belhouari. - Advanced Materials Research. - 2015. - Vol. 1105. - P. 41-45.

4. Suner $S$. Ultra high molecular weight polyethylene/graphene oxide nanocomposites: Thermal, mechanical and wettability characterisation / S. Suner, R. Joffe, J.L.Tipper, N. Emami. Composites Part B. - 2015. - Vol. 78. - P. 185-191.

5. Damodaran S. Monitoring the effect of chlorine on the ageing of polypropylene pipes by infrared microscopy / Subin Damodaran, Tobias Schuster, Karsten Rode, Abhishek Sanoria, Robert Brüll, Mirko Wenzel, Martin Bastian. - Polymer Degradation and Stability. - 2015. - Vol. 66 P. 7-19.

6. Agari Y. Thermal Conductivity of a Polymer Composite Filled with Mixtures of Particles / Y. Agari, M. Tanaka, S. Nagai. - Journal of Applied Polymer Science. - 1987. - Vol. 34. - P. 1429-1437.

7. Lin C. Modeling and analysis of synergistic effect in thermal conductivity enhancement of polymer composites with hybrid filler / Lin Chen, Ying-Ying Sun, Jun Lin, Xiao-Ze Du, Gao-Sheng Wei, Shao-Jian He, Sergei Nazarenko. - International Journal of Heat and Mass Transfer. - 2015. - Vol. 81. - P. 457-464.

8. Shen $M$. Thermal conductivity model of filled polymer composites / Ming-xia Shen, Yin-xin Cui, Jing He, and Yao-ming Zhang. - International Journal of Minerals, Metallurgy and Materials. - 2011. - Vol. 18, №5. - P. 623-631.

9. Kirkpatrick S. Percolation and Conduction / Scott Kirkpatrick. - Reviews of modern physics. 1973. - Vol. 45, №4. - P. 574-585.

10. McLachlan D.S. The correct modelling of the second order terms of the complex ACconductivity results for continuum percolation media, using a single phenomenological equation / D.S. McLachlan*, C. Chiteme, W.D. Heiss, Junjie Wu. Physica B. - 2003. - Vol. 338. - P. 256-260.

11. Dolinskiy A.A. Thermophysical properties 
of polymer micro- and nanocomposites based N.M. Fialko, R.V.Dinzhos, R.A. Navrodskaya // on polycarbonate / A.A. Dolinskiy, N.M. Fialko, Industrial heat engineering. - 2015 . - № 4. - P. 5-13. R.V. Dinzhos, R.A. Navrodskaya // Industrial heat (Rus) engineering. - 2015. - №2. - P.12-19. (Rus)

13. Katsevman M. Application of concentrates

12. Dolinskiy A.A. Influence of methods of preparation of polymeric micro- and nanocomposites on their thermophysical properties / A.A. Dolinskiy, of mineral fillers in the production of plastic pipes / M. Katsevman, S. Kiselyov, I. Ayzinsov. - Polymeric pipes. - 2011. - Vol. 31. - P. 31-37.

Получено 02.11.2015

Received 02.11.2015 